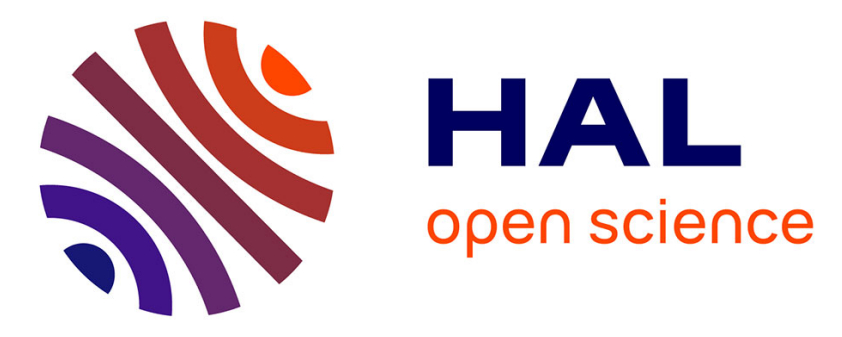

\title{
Nonnegative CP decomposition of multiangle hyperspectral data: a case study on CRISM observations of martian icy surface
}

Miguel Angel Veganzones, Sylvain Douté, Jérémy E Cohen, Rodrigo Cabral Farias, Jocelyn Chanussot, Pierre Comon

\section{To cite this version:}

Miguel Angel Veganzones, Sylvain Douté, Jérémy E Cohen, Rodrigo Cabral Farias, Jocelyn Chanussot, et al.. Nonnegative CP decomposition of multiangle hyperspectral data: a case study on CRISM observations of martian icy surface. WHISPERS 2016 - 8th Workshop on Hyperspectral Image and Signal Processing: Evolution in Remote Sensing, Aug 2016, Los Angeles, CA, United States. hal01382360

\section{HAL Id: hal-01382360 https://hal.science/hal-01382360}

Submitted on 6 Nov 2016

HAL is a multi-disciplinary open access archive for the deposit and dissemination of scientific research documents, whether they are published or not. The documents may come from teaching and research institutions in France or abroad, or from public or private research centers.
L'archive ouverte pluridisciplinaire HAL, est destinée au dépôt et à la diffusion de documents scientifiques de niveau recherche, publiés ou non, émanant des établissements d'enseignement et de recherche français ou étrangers, des laboratoires publics ou privés. 


\title{
NONNEGATIVE CP DECOMPOSITION OF MULTIANGLE HYPERSPECTRAL DATA: A CASE STUDY ON CRISM OBSERVATIONS OF MARTIAN ICY SURFACE
}

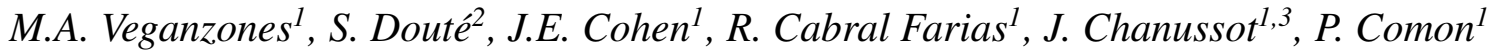 \\ ${ }^{1}$ CNRS, Gipsa-Lab, Univ. Grenoble Alpes, F-38000 Grenoble, France \\ ${ }^{2}$ Institut de Planétologie et d'Astrophysique de Grenoble, CNRS, F-38041 Grenoble \\ ${ }^{3}$ Faculty of Electrical and Computer Engineering, University of Iceland, Reykjavik, Iceland
}

\begin{abstract}
The Compact Reconnaissance Imaging Spectrometer for Mars (CRISM) sensor aboard the Mars Reconnaissance Orbiter takes hyperspectral multi-angle acquisitions of Martian surface from the top of the atmosphere (TOA) on visible and infrared wavelengths. The Multiangle Approach for Retrieval of Surface Reflectance from CRISM Observations (MARS-ReCO) defined an innovative TOA radiance model and inversion scheme aimed at correcting for aerosols effects taking advantage of the near-simultaneous multiangle CRISM observations. Here, we aim to provide validation evidence of MARS-ReCO by unmixing the estimated multi-angle bidirectional reflectance (BRF) from highly reflective and anisotropic icy surfaces at high latitudes with grazing illumination, using a nonnegative $\mathrm{CP}$ decomposition. Obtained results are in accordance with other complementary studies, which compose a collaboration effort to validate MARS-ReCO through the cross-validation of different techniques in the absence of ground truth.
\end{abstract}

Index Terms - CRISM mission, multi-angle hyperspectral, MARS-ReCO, Canonical-Polyadic, nonnegative tensor decomposition.

\section{INTRODUCTION}

The Compact Reconnaissance Imaging Spectrometer for Mars (CRISM) sensor aboard the Mars Reconnaissance Orbiter takes hyperspectral acquisitions of Martian surface from the top of the atmosphere (TOA) using 11 viewing angles in 437 spectral bands, on visible and infrared wavelengths. The CRISM experiment is an invaluable source of information related to the scattering of solar light by surface materials and atmospheric aerosols. However, the extraction of surface bidirectional reflectance (BRF) is especially challenging, due to the strong effects of aerosols in Martian atmosphere. The Multiangle Approach for Retrieval of Surface Reflectance from CRISM Observations (MARS-ReCO) [1, 2] defined an innovative TOA radiance model and inversion scheme aimed at correcting for aerosols effects taking advantage of the near-simultaneous multi-angle CRISM observations. MARS-ReCO retrieves the aerosol optical depth (AOD) and the BRF of surface materials as a function of wavelength.

The validation of MARS-ReCO has been solely performed on the base of controlled synthetic data [1] and by comparison with in situ reflectance measurements by the Martian Exploration Rovers

This work is supported by the European Research Council under the European Communitys Seventh Framework Programme FP7/2007-2013 Grant Agreement no. 320594, "DECODA". Also, by a contract with PNTS, ANR (AAP Blanc 2012 Inter II - SIMI 5) and a grant from Label OSUG@2020 (Investissements davenir ANR10 LABX56).
(MERs) [3]. This validation was restricted to moderately anisotropic mineral surfaces. Here, we aim to provide supporting evidence by performing a spectral unmixing (SU) analysis of MARS-ReCO BRF estimates from CRISM multiangle observations of highly reflective and anisotropic icy surfaces at high latitudes with grazing illumination. This is part of a collaboration effort to validate MARS-ReCO through the cross-validation of different techniques in the absence of ground truth [4].

Spectral unmixing (SU) is one of the most studied topics in hyperspectral image analysis. The goal of SU techniques is to estimate the spectral signatures of the materials present in the image (endmembers) and their spatial distributions (fractional abundances). Usually, a linear mixing model (LMM) is considered:

$$
\boldsymbol{X}=\boldsymbol{\Phi} \boldsymbol{S}^{\top}+\boldsymbol{E},
$$

where $S \in \mathbb{R}_{+}^{L \times R}$ denotes the matrix of endmembers, each of the $R$ columns representing the spectral signature of a given macroscopic material, $\boldsymbol{\Phi} \in \mathbb{R}_{+}^{N \times R}$ denotes the matrix of fractional abundances corresponding to each of the $R$ endmembers; and $\boldsymbol{E} \in \mathbb{R}^{N \times L}$ denotes additive noise.

In $[5,6]$ we proposed to exploit the hyperspectral multiangle information by representing the hyperspectral multiangle acquisitions as a nonnegative tensor, $\mathcal{X} \in \mathbb{R}_{+}^{N \times L \times T}$, with $N$ pixels, $L$ wavelengths and $T$ angles of acquisition. Tensor analysis [7] (a.k.a. multiway or multiarray analysis) techniques, and specifically the Canonical Polyadic (CP) model [8], sometimes coined Candecomp/Parafac [9], can be used to decompose the multiangle tensor, $\mathcal{X}$, into a multilinear combination of $R$ nonnegative factor matrices $\boldsymbol{A}, \boldsymbol{B}$ and $\boldsymbol{C}$, of size $N \times R, L \times R$ and $T \times R$, respectively:

$$
\mathcal{X}_{i j k}=\sum_{r=1}^{R} A_{i r} B_{j r} C_{k r},
$$

where the $\boldsymbol{A}, \boldsymbol{B}$ and $\boldsymbol{C}$ factors are associated with estimations of the fractional abundances, endmembers and angular anisotropies of the materials in the scene. Thus, the nonnegative $\mathrm{CP}$ decomposition can be understood as a multilinear spectral unmixing, exploiting the additional angular information.

The rank of tensor $\mathcal{X}$ is defined as the minimal number $R$ of terms necessary for the equality above to hold exactly. A shorter expression can be employed to denote the decomposition in (2) using a diagonal tensor of ones, $\mathcal{I}$, of size $R \times R \times R$, i.e., $(\boldsymbol{A} \otimes \boldsymbol{B} \otimes \boldsymbol{C}) \mathcal{I}$, where $\otimes$ is a tensor product. In practice, the data tensor is subject to modeling errors or measurement noise, and it is convenient to find its best rank- $R$ approximation by minimizing an objective function 


\begin{tabular}{|l|l|l|l|l|}
\hline CRISM ID & Ls $^{1}$ & Incidence & Phase range & $\mathrm{AOD}^{2}$ \\
\hline FRT144E9 & $320^{\circ}$ & $72^{\circ}$ & $35-128^{\circ}$ & 0.62 \\
\hline
\end{tabular}

Table 1. Information of the CRISM observation used in the study case.

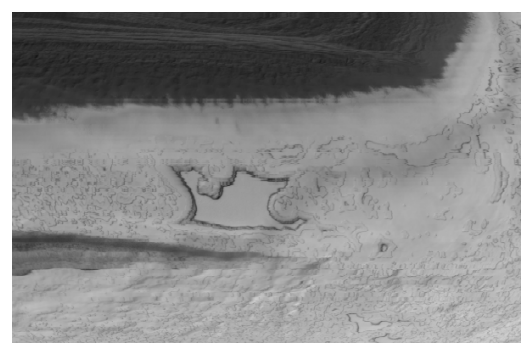

Fig. 3. High-resolution gray scale image of the study case scene taken by CRISM sensor at nadir angle.

of the form

$$
\Upsilon(\boldsymbol{A}, \boldsymbol{B}, \boldsymbol{C})=\|\mathcal{X}-(\boldsymbol{A} \otimes \boldsymbol{B} \otimes \boldsymbol{C}) \mathcal{I}\|^{2},
$$

for some well chosen norm, instead of solving the exact CP decomposition (2). It is known that tensors of order 3 or larger do not always admit a best rank- $R$ approximate, when $R>1$, especially in $\mathbb{R}$ or $\mathbb{C}$. But fortunately, it has been shown in [10] that such obstacle does no exist for nonnegative tensors, and that the problem is well-posed in $\mathbb{R}^{+}$: best lower rank nonnegative approximates always exist and are generically unique under mild conditions on $R$ [11].

Here, we study the application of the nonnegative CP decomposition method to unmix a multiangle BRF data cube extracted by MARS-ReCO from a challenging area of interest in the outskirts of the south permanent polar cap in Mars.

\subsection{Data}

\section{DATA AND METHODS}

The data set is a multi-angle CRISM acquisition in the outskirts of the south permanent polar cap in Mars. The analysis here is restricted to the visible channel (VIS) of the instrument $(0.4-1.0 \mu \mathrm{m})$. The scene features regions of submiating icy $\mathrm{CO}_{2}$, revealing $\mathrm{H}_{2} \mathrm{O}$ frost and defrosted terrains. The topography of the region is moderate with maximum slopes of $10 \%$. No ground truth is available for this region. Table 1 gives information about the data set, and Fig. 3 depicts a high-resolution gray scale image of the scene taken at nadir. In order to facilitate the simultaneous processing of the multiangle TOA reflectance factor of CRISM observation, the spectra corresponding to the eleven view angles are rearranged in a common geographical grid of super-pixels. Each spatial bin is approximately 200 meters in size [2]. Super pixels are stacked in one way, so that after preprocessing the resulting data is a third order tensor with super pixels, wavelengths and angles as diversities.

\subsection{Nonnegative $\mathrm{CP}$ decomposition}

Given a nonnegative tensor, $\mathcal{X} \in \mathbb{R}_{+}^{N \times L \times T}$, its approximation by a nonnegative $\mathrm{CP}$ decomposition is formulated as:

$$
\begin{gathered}
\operatorname{argmin}\|\mathcal{X}-(\boldsymbol{A} \otimes \boldsymbol{B} \otimes \boldsymbol{C}) \mathcal{I}\|_{F}^{2} \\
\text { w.r.t. } \boldsymbol{A}, \boldsymbol{B}, \boldsymbol{C} \\
\text { s.t. } \boldsymbol{A} \succeq 0, \boldsymbol{B} \succeq 0, \boldsymbol{C} \succeq 0,
\end{gathered}
$$

where $\|\cdot\|_{F}$ denotes the Frobenius norm, and inequality $\succeq$ applies entry-wise. This problem is highly non-convex. The most commonly used method for nonnegative $\mathrm{CP}$ decomposition is alternating nonnegative least squares (ANLS) [12], where the cost function is minimized in an alternating way for each factor $(\boldsymbol{A}, \boldsymbol{B}$ or $\boldsymbol{C})$ while the others are fixed.

When the data tensor is large as in hyperspectral multiangle data, where the number of pixels is usually larger than $10^{6}$, the workhorse techniques described briefly above can fail to handle all the data within the memory of the computer, or can converge very slowly. An approach to handle large tensor decomposition is through the use of compression. The general idea is that the original tensor $\mathcal{X}$ can be equivalently represented by a compressed version of it, $\mathcal{X}_{c}$, with reduced dimensions $N_{c} \times L_{c} \times T_{c}$. The compressed tensor is then decomposed by minimizing

$$
\begin{gathered}
\Upsilon=\left\|\boldsymbol{X}_{c}-\left(\boldsymbol{A}_{c} \otimes \boldsymbol{B}_{c} \otimes \boldsymbol{C}_{c}\right) \mathcal{I}\right\|_{F}^{2} \\
\text { w.r.t. } \boldsymbol{A}_{c}, \boldsymbol{B}_{c}, \boldsymbol{C}_{c},
\end{gathered}
$$

where $\boldsymbol{A}_{c}, \boldsymbol{B}_{c}, \boldsymbol{C}_{c}$ are compressed versions of the original factor matrices. Note that, after the compressed factors are obtained, a decompression operation must carried out to recover nonnegative factors in the original dimensions.

Authors in [13] provide two algorithms, a compressed conjugate gradient (CCG) and a Projected and Compressed Alternating Least Squares (ProCo-ALS) to solve (5) when the compression is done using an approximate High Order Singular Value Decomposition (HOSVD):

$$
\boldsymbol{\mathcal { X }}_{i j k} \approx \sum_{l m n}^{N_{c}, L_{c}, T_{c}} \boldsymbol{U}_{i l} \boldsymbol{V}_{j m} \boldsymbol{W}_{k n}\left[\boldsymbol{\mathcal { X }}_{c}\right]_{i j k},
$$

or more compactly $\mathcal{X} \approx(\boldsymbol{U} \otimes \boldsymbol{V} \otimes \boldsymbol{W}) \boldsymbol{\mathcal { X }}_{c}$, where $\boldsymbol{U}, \boldsymbol{V}$ and $\boldsymbol{W}$ are matrices with orthogonal unit-norm columns. These algorithms solve the optimization problem defined in (5) enforcing the nonnegativity of the factors in the unconstrained domain:

$$
\boldsymbol{A} \approx \boldsymbol{U} \boldsymbol{A}_{c} \succeq 0, \quad \boldsymbol{B} \approx \boldsymbol{V} \boldsymbol{B}_{c} \succeq 0, \quad \boldsymbol{C} \approx \boldsymbol{W} \boldsymbol{C}_{c} \succeq 0 .
$$

Here, we make use of a modified version of ProCo-ALS to include the sum-to-one physical constraint on abundances, usually employed in spectral unmixing:

$$
A 1_{R}=\mathbf{1}_{N} .
$$

The modified ProCo-ALS algorithm works by projecting $\boldsymbol{A}$ onto the unitary simplex instead of projecting it onto the nonnegative orthant using the solution to the waterfilling problem.

\section{EXPERIMENTAL METHODOLOGY AND RESULTS}

We run two sets of 20 Monte Carlo runs of the modified ProCo-ALS CP decomposition over the Mars reflectance tensor representation, using two tensor rank values, $R=\{3,4\}$. Those superpixels of the reflectance tensor for which there were missing values were removed, so the number of tensor superpixels was $N=1633$. The dimensions of the compressed tensor were set to $N_{c}=550, L_{c}=50$ and $T_{c}=11$, so only the spatial way was slightly compressed in approximately a $1 / 3$ ratio. For each ProCo-ALS run, we randomly initialized the factors $\boldsymbol{A}, \boldsymbol{B}, \boldsymbol{C}$ to nonnegative matrices, ensuring that the spectral factor, $\boldsymbol{B}$, is normalized and, that the spatial factor, $\boldsymbol{A}$, holds the sum-to-one constraint. Among the 20 runs, we kept the one maximizing the volume of the simplex formed by the columns 


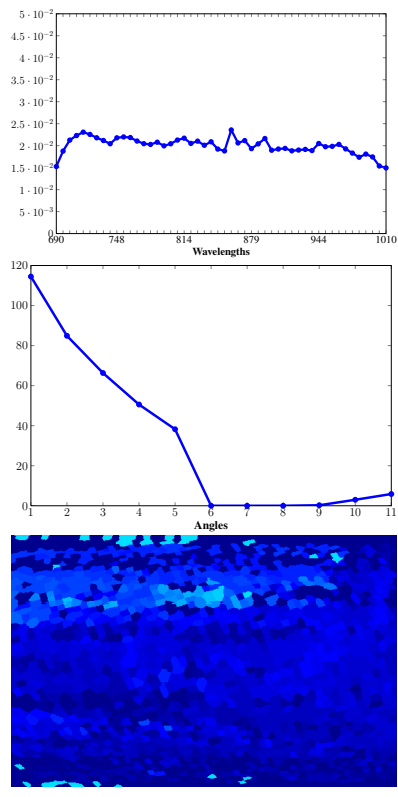

First component
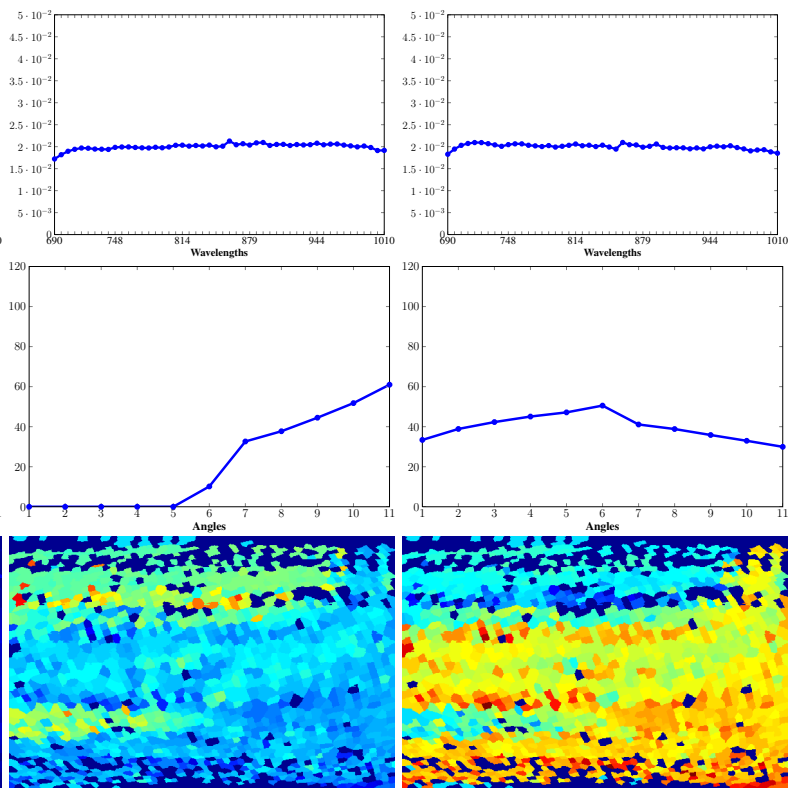

Second component

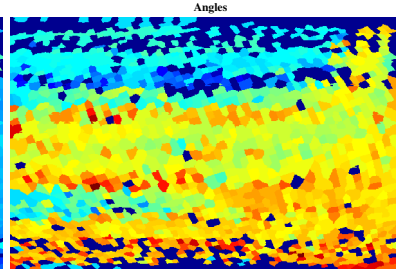

Third component

Fig. 1. Best run ontained by the modified ProCo-ALS with $R=3$ : (top) Spectral factor, $\hat{\boldsymbol{B}}$, (mid) Angle factor, $\hat{\boldsymbol{C}}$, and (bottom) Spatial factor, $\hat{\boldsymbol{A}}$.
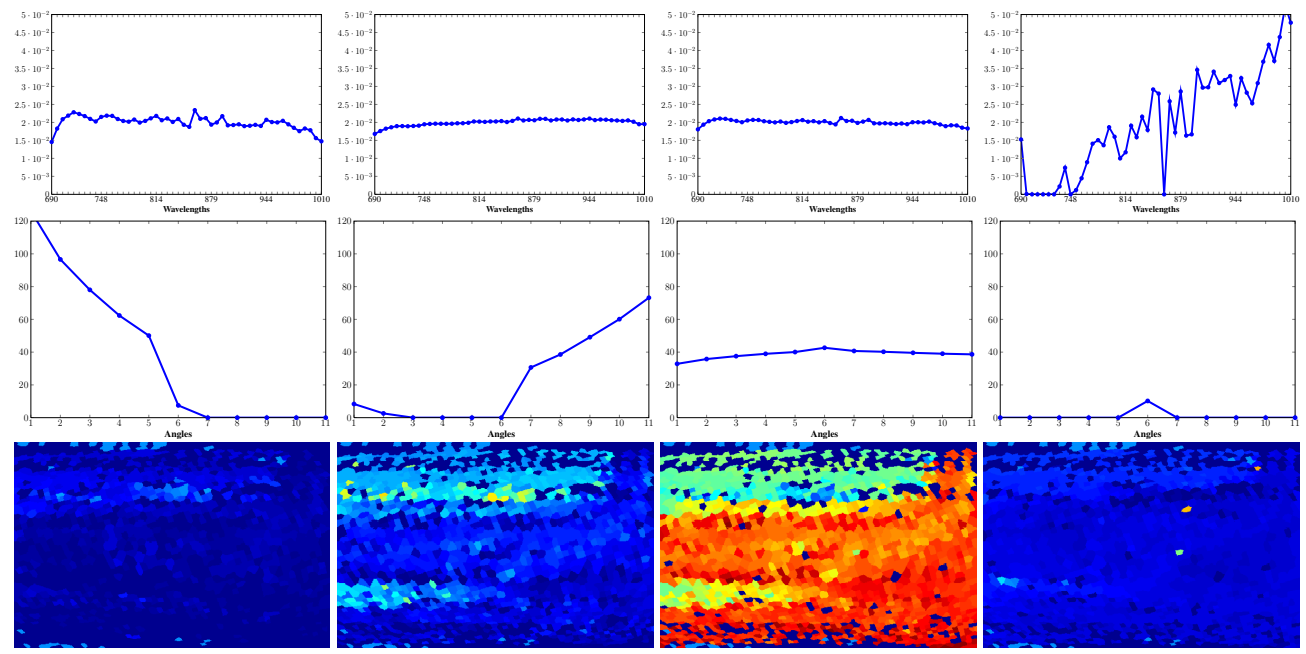

Fist component

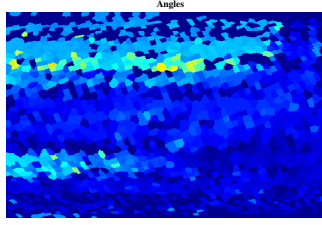

Second component

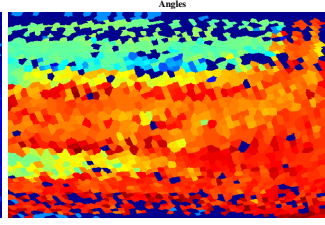

Third component
Fourth component

Fig. 2. Best run ontained by the modified ProCo-ALS with $R=4$ : (top) Spectral factor, $\hat{\boldsymbol{B}}$, (mid) Angle factor, $\hat{\boldsymbol{C}}$, and (bottom) Spatial factor, $\hat{\boldsymbol{A}}$.

of the spectral factor matrix, $V(\boldsymbol{B})[14]$ :

$$
V(\boldsymbol{B})=\frac{1}{R-1 !}\left|\operatorname{det}\left(\begin{array}{ccc}
1 & \ldots & 1 \\
\boldsymbol{b}_{1} & \ldots & \boldsymbol{b}_{R}
\end{array}\right)\right| .
$$

Figs. 1 and 2 show the factors estimated by the best run according to (9) for $R=3$ and $R=4$, respectively. The only significant differences between the two solutions are: (i) the angle factors of the first and second components have a low tail appearing at last angle indices of the first component for $R=3$, and at the first angle indices of the second component for $R=4$; (ii) the spatial factors of the second and third components for rank $R=4$ are shifted to more extreme values w.r.t. those obtained for $R=3$; and, (iii) the additional component estimated for $R=4$ seems to be an artefact affecting a few superpixels in the nadir angle $(T=6)$.

The VIS range offers no distinct features to distinguish the materials present on the image, because of the relative flat behaviour of the spectral factors. On the contrary, the angular factors give information that can be physically interpreted. The first two compo- 


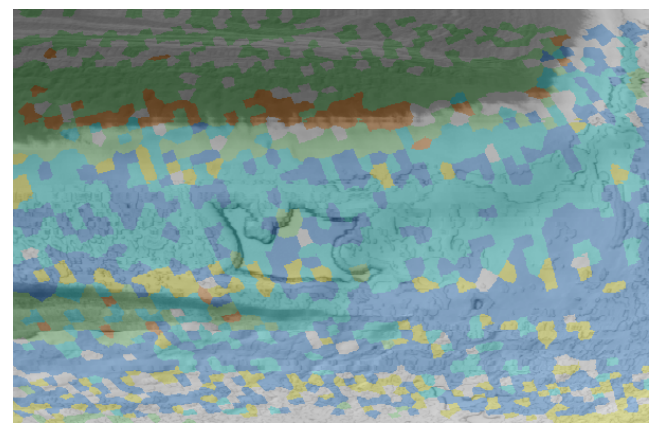

(a)

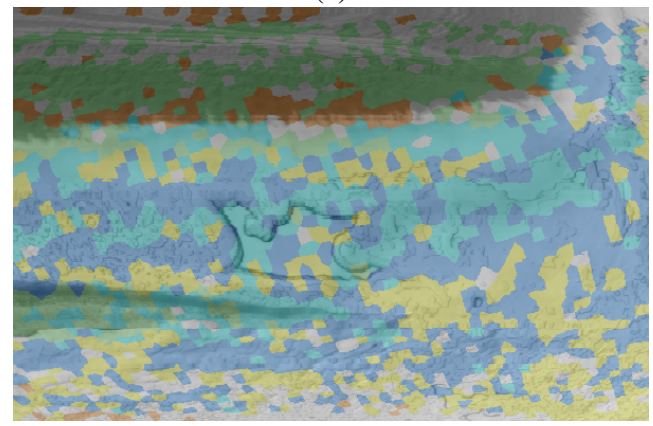

(b)

Fig. 4. Results of a k-mean clustering $(k=5)$ over the spatial factor estimated by ProCo-ALS with (a) $R=3$ and (b) $R=4$. Figures depict the clustering maps in transparent colors over a high resolution grayscale image of the scene.

nents present high anisotropic behaviours. The first one displays a strong backward scattering lobe (high values at low phase angles, $T=1 \ldots 5$ ), associated with the presence of extended shadows (sun low on the horizon) at the sub-pixel scale. The second one has a marked forward-scattering scattering lobe (high values at high phase angles, $T=8 \ldots 11$ ), linked with volume scattering within the surface materials. Finally, the third component shows much less anisotropic behaviour and, upon mixing, likely controls the overall level of the BRF as a function of geometry, i.e. the intrinsic albedo.

Fig. 5 shows the results of a k-means clustering over the spatial factors obtained by ProCo-ALS for a number of clusters $k=5$. The fourth component estimated by ProCo-ALS with $R=4$ has been discarded in the analysis. Results obtained by setting $k=5$ present a high degree of internal coherence..According to Fig. 5, the dark unit of the nadir image (top and middle-left strips) could be decomposed into two clusters (red and green), while the brightest unit of the nadir image consists in three distinguishable clusters (blue, cyan and yellow). The latter three clusters share the high presence of the third component where dominates highly reflective $\mathrm{CO}_{2}$ ice but with a moderately anisotropic behaviour: smaller relative proportions of the other two materials (with high anisotropies). The difference among these segmented areas appears to be related to the topography of the region and its relation to the shadowing effects. The dark unit, composed of two distinct areas, presents a higher relative abundance of the second component (backward scattering) but a varying abundance of the third component (moderate for the fourth cluster (red) but high for the fifth (green). Also, the former cluster presents a higher abundance of first component relative to the lat-

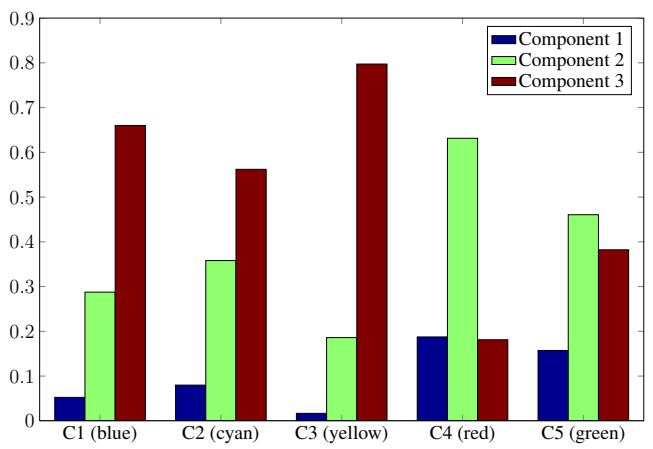

(c)

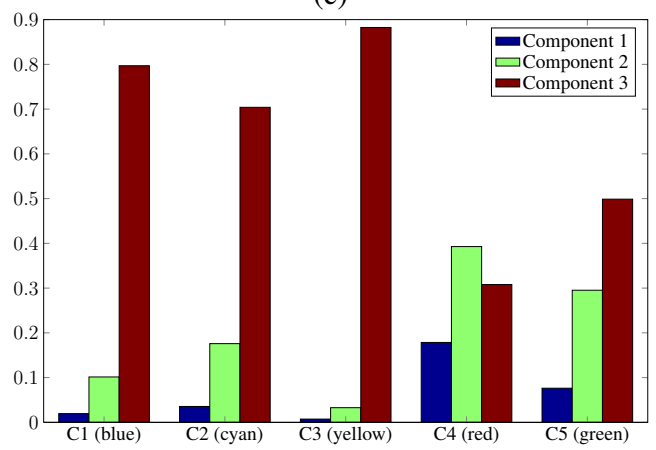

(d)

Fig. 5. Results of a k-mean clustering $(k=5)$ over the spatial factor estimated by ProCo-ALS with (a) $R=3$ and (b) $R=4$. Bar plots show the centroids of the clusters in terms of the three components estimated by ProCo-ALS (the fourth component for $R=4$ has been discarded in the analysis).

ter cluster. The green cluster corresponds to a mixture of dust and minute amount of water ice (mostly defrosted terrains), while the red cluster corresponds to compositional transition zones with a high concentration of $\mathrm{H}_{2} \mathrm{O}$ frost. These results are in accordance with the ones obtained from a temporal series of the studied area in [4].

As expected, the results of the unmixing algorithm for the spectral way are hard to interpret since in the visible range, materials expected to be found on the surface of Mars present no discriminative features. Moreover in our analysis, the recovered spectra are almost collinear, suggesting the spectral diversity may not be necessary. Further analysis will be carried out to try other low rank unmixing methods like Nonnegative Matrix Factorization. Coupling present results with an analysis of infrared data for which materials should have distinguishable spectra should also prove interesting.

\section{CONCLUSIONS}

We have studied the unmixing of a multiangle BRF data cube extracted by MARS-ReCO from a challenging area of interest in the outskirts of the south permanent polar cap in Mars, by means of nonnegative $\mathrm{CP}$ decomposition. This work is part of a collaborative effort to validate MARS-ReCO through the cross-validation of different techniques in absence of ground truth. Obtained results are in accordance with other studies involved in the MARS-ReCO validation project, supporting the estimations of MARS-ReCO and the use of nonnegative $\mathrm{CP}$ decompositions to unmix multiangle hyperspectral data. 


\section{REFERENCES}

[1] X. Ceamanos, S. Douté, J. Fernando, F. Schmidt, P. Pinet, and A. Lyapustin, "Surface reflectance of Mars observed by CRISM/MRO: 1. multi-angle approach for retrieval of surface reflectance from CRISM observations (MARS-ReCO)," J. Geophys. Research: Planets, vol. 118, pp. 514-533, 2013.

[2] S. Douté and X. Ceamanos, "Multi-angle approach for coherent retrieval of surface reflectance and aerosol optical depth from CRISM observations," in Int. Symp. Geoscience and Remote Sensing (IGARSS), July 2015, pp. 1-4.

[3] J. Fernando, F. Schmidt, X. Ceamanos, P. Pinet, S. Douté, and Y. Daydou, "Surface reflectance of Mars observed by CRISM/MRO: 2. estimation of surface photometric properties in gusev crater and meridiani planum," J. Geophys. Research: Planets, vol. 118, no. 3, pp. 534-559, 2013.

[4] S. Douté and C. Pilorget, "Testing the multi-angle approach for retrieval of surface reflectance from crism observations on martian icy surfaces," in 8 rd Workshop on Hyperspectral Image and Signal Processing: Evolution in Remote Sensing (WHISPERS), 2016, Submitted.

[5] M. A. Veganzones, J. E. Cohen, R. Cabral Farias, R. Marrero, J. Chanussot, and P. Comon, "Multilinear spectral unmixing of hyperspectral multiangle images," in 23rd European Sig. Proc. Conf. (EUSIPCO), Aug 2015, pp. 744-748.

[6] M. Veganzones, J. E. Cohen, R. Cabral Farias, J. Chanussot, and P. Comon, "Nonnegative tensor CP decomposition of hyperspectral data," IEEE Trans. Geoscience Remote Sensing, vol. 54, no. 5, pp. 2577-2588, May 2016.

[7] P. Comon, "Tensors : A brief introduction," IEEE Sig. Proc. Mag., vol. 31, no. 3, pp. 44-53, May 2014.

[8] F. L. Hitchcock, "The expression of a tensor or a polyadic as a sum of products," J. Math. Physics, vol. 6, pp. 165-189, 1927.

[9] H. A. L. Kiers, "Towards a standardized notation and terminology in multiway analysis," J. Chemometrics, vol. 14, pp. 105-122, 2000.

[10] L.-H. Lim and P. Comon, "Nonnegative approximations of nonnegative tensors," J. Chemometrics, vol. 23, no. 7-8, pp. 432-441, 2009.

[11] Y. Qi, P. Comon, and L. H. Lim, "Uniqueness of non-negative tensor approximations," IEEE Trans. Inf. Theory, vol. 62, no. 4, pp. 2170-2183, 2016, arXiv:1410.8129.

[12] A. Cichocki, R. Zdunek, A. H. Phan, and S.-I. Amari, Nonnegative matrix and tensor factorizations, Wiley, 2009.

[13] J.E. Cohen, R.C. Farias, and P. Comon, "Fast decomposition of large nonnegative tensors," IEEE Sig. Proc. Letters, vol. 22, no. 7, pp. 862-866, July 2015.

[14] L. Miao and H. Qi, "Endmember extraction from highly mixed data using minimum volume constrained nonnegative matrix factorization," IEEE Trans. Geoscience Remote Sensing, vol. 45, no. 3, pp. 765-777, March 2007. 